\title{
The Intestinal Microbiota in Health and Disease
}

\author{
Vincent B. Young, MD, PhD \\ Department of Internal Medicine/Division of Infectious Diseases Department of Microbiology \& \\ Immunology, The University of Michigan, Ann Arbor, MI, 48109, USA
}

\begin{abstract}
Purpose of review-The indigenous gut microbiota has been shown to be a key player in maintaining gastrointestinal homeostasis. This review discusses some of the recent work that reveals how the gut microbiome helps establish and protect intestinal health and how disturbances in this microbial community can lead to disease states.
\end{abstract}

Recent findings-The use of culture-independent methods has greatly improved our ability to determine the structure and function of the gut microbiome. The gut microbiota has critical interactions with the host immune system and metabolism with bilateral influences shaping both the host and the microbiome. Alterations in the gut microbiome are associated with a variety of disease states but we are only now beginning to understand the mechanisms by which this occurs.

Summary-Understanding how the gut microbiome contributes to intestinal health should lead to novel preventative strategies and therapies for a variety of gastrointestinal conditions.

\section{Keywords}

Microbiome; host-microbe interactions; microbiota

\section{Introduction}

The realization that the complex communities of microbes that inhabit the human body play a crucial role in the health of the host has led to an explosion of research activity.

Historically, studies of the microbiota that inhabit the gastrointestinal tract have been most numerous and this review will discuss some of the recent literature that has increased our understanding of the complex interaction between gut microbial communities and their mammalian hosts.

Much of the progress in studying various aspects of the human microbiome has been driven by technological advances in sequencing and their application to the culture-independent analysis of the structure and function of complex microbial communities. We will not attempt to review these techniques in any detail. Readers who would like more information regarding the technical aspects of microbiome research, including data generation and analysis, are referred to a number of recent reviews (1-3).

\section{The Intestinal Microbiota and Gut Homeostasis}

Prior to embarking on a discussion of recent work correlating changes in the microbiome with disease states, it is important to review some of the recent progress that has been made in understanding the normal interactions between the gut microbiome and the host.

\footnotetext{
*Corresponding author: Vincent B. Young, MD, PhD, The University of Michigan, 5510A MSRB I, SPC 5680, $1150 \mathrm{~W}$. Medical Center Dr. Ann Arbor, MI 48109-5623, Office: 734-764-2237, Fax: 734-763-4168, youngvi@umich.edu.
} 


\section{Novel Insights into the "Normal Gut Microbiome"}

Globally, there are multiple initiatives that are attempting to understand the interaction between the gut microbiota and the host. The NIH-funded Human Microbiome Project (4) and the International MetaHIT initiative have invested significant effort in delineating the normal structure and function of the gut microbiota. Some of the efforts from these and other initiatives will be reviewed in this section. The initial discussion will focus on efforts to define the normal structure of the microbiome. However, to draw an analogy, these structural studies are meant to better understand the "anatomy" of the microbiome. As anatomy is only a beginning as to obtaining a complete understanding of an organism, we will then turn to a discussion of studies that are looking at the "physiology" of the microbiome and focus on the functional aspects of the gut microbial community.

\section{Community Structure of the Gut Microbiota}

A large study was published by the international MetaHIT consortium. This group examined 22 metagenomes generated from fecal samples and combined these data with previously published data sets. A total of 39 individuals were part of this analysis. Examination of this data set indicated that the microbial communities segregated into 3 distinct clusters, which the authors referred to as "enterotypes" $\left(5^{* *}\right)$. The individuals in this study represented a combination of individuals from Europe, Japan and the United States. The 3 enterotypes were not specific to a particular geographic region. Since the data in this study was metagenomic, they could be analyzed for both phylogenetic and functional signals. The existence of three enterotypes was supported by both phylogenetic and functional analysis. Each enterotypes had specific aspects of species and functional composition that were unique compared to the other two. It remains to be seen if these enterotypes will be seen in additional analyses that are currently ongoing. Furthermore, additional work will need to be done to determine what factors drive the segregation of communities into these enterotypes.

Related to the question of host factors that may drive the community structure of the gut microbiota, a study was conducted to identify host genetic determinants that influence the microbiome $(6 *)$. This study employed a moderately advanced intercrossed mouse line and examined fecal samples from 645 animals. The microbiome was analyzed by pyrosequencing of the $16 \mathrm{~S}$ gene. Variation in the composition of the gut microbiota was analyzed for influence of the maternal environment and genotype. In concordance with previous studies, lineage (i.e. litter and cohort) had a significant effect on microbiota composition, but host genetics also explained a significant proportion of the variation. Quantitative trait loci (QTL) analysis, based on 530 single nucleotide polymorphisms, was used to assess the degree to which genotype influenced microbiota composition. 18 QTL were identified that had significant linkage to the relative abundances of specific microbial taxa. The authors interpret their data as suggesting that the composition of the gut microbiota behaves as a complex polygenic trait. This should set the stage for future studies that will attempt to define the mechanisms that actually drive the assembly and structure of the microbiome.

Although genetics can be a key driver of the community structure of the microbiome, it is clear that exogenous factors can also play a significant role. One of these exogenous factors that has received a great deal of attention is the administration of antibiotics. Although it is clear that the gut microbiota is dramatically changed under the pressure of antibiotic administration (7), is not known how well the community can recover from such a disturbance. This question about the ecologic resilience of the gut community was addressed in a human volunteer study where 3 individuals received 2 courses of antibiotic ciprofloxacin and were followed for nearly a year $\left(8^{*}\right)$. In spite of the fact that none of the 
patients developed symptoms while on the antibiotic therapy and had no change otherwise in their health, there was a dramatic shift in the structure of the gut microbiota. Furthermore recovery of the gut microbial community following the cessation of the 5-day course of antibiotics was incomplete and varied among the 3 individuals in this study.

\section{Metabolic Function of the Gut Microbiota}

With the above studies as examples it is clear that we are gaining a deeper understanding of the structure, assembly and dynamics of the gut microbiota. However in and of themselves, these studies do not provide a link between community structure and function.

Understanding structure/function relationships in the microbiome is an active area of research (3). It is important to realize that studies that focus solely on determining structure of the microbiome (for example, via the retrieval of $16 \mathrm{~S}$ sequences) will be unable to address key functional aspects of the community. Communities with structures that are quite distinct may nevertheless have similar functions, with taxonomically distinct organisms carrying out interchangeable roles in the community. Therefore, a fuller understanding of the function of a given community may best be accomplished by studies that combine structural analysis with direct measurement of the functions being carried out by the community.

One of the key functions that have been ascribed to the gut microbiota concerns the metabolic functions that this community can carry out. When considered as a discrete unit, the combined coding capacity of the microbiome (referred to as the metagenome) exceeds that of the host. The gut microbiota can metabolize material ingested by the host as well as products of the hosts on metabolism. In turn, products of the microbial metabolism can be further converted by the host. Thus the metabolite profile in the gut results from a combination of host metabolism, microbial and mammalian-microbial cometabolism. Differences in the overall intestinal metabolism can be seen in germ-free animals compared to animals with a complete microbiota. Antibiotic administration, presumably through alteration of the indigenous microbiota, can also have a profound effect on the metabolic profile of the gut and the entire animal. Treatment of mice with streptomycin was found to affect the levels of over $87 \%$ of all metabolites detected in feces $(9 *)$. Multiple metabolic pathways were affected including the metabolism of bile acids, inflammatory lipids and steroid hormones. Such effects are not simply confined to the intestinal tract as other investigators who treated rats with a combination of streptomycin penicillin noted alterations in the metabolic profile of not only the feces but also the urine, indicating that systemic effects on host metabolism also took place (10*).

Although global effects on host metabolism are likely to arise from changes in the community structure of the gut microbiota, the interaction between this microbial community and the intestinal tract is likely to be the most dramatic. One group demonstrated that the microbiota, has a dramatic effect on energy homeostasis in the colon compared to other host tissues $\left(11^{* *}\right)$. The authors demonstrated that this tissue specificity was due primarily to the production of the short chain fatty acid butyrate by the microbiota. Butyrate serves as the primary energy source for colonocytes and colonocytes from germ-free mice were shown to be in a energy-deprived state. Interestingly, this energy deprivation led to autophagy in these colonocytes, whereby the cells degraded their own cellular components in an attempt to maintain energy homeostasis. The host on the other hand, can also use metabolic input into the gastrointestinal ecosystem to shape the microbiota. Mice colonized with human feces establish a complex microbiota (12). Changes in the diet from a low-fat, plants-rich diet to a high-fat, high-sugar diet resulted in rapid changes in the gut microbial community. The change in community structure was accompanied by a change in the metabolic potential of this community reflecting a functional change to deal with the change 
in diet. Another example of the role diet plays in shaping the intestinal microbiota comes from studies that show how the presence of specific oligosaccharides in human milk can shape the gut community (13). Such studies indicate the evolutionary development of the means by which mammals can selectively nourish appropriate microbial symbionts early in infant development.

\section{Interactions of the Gut Microbiota and the Host Immune System}

In addition to the complex interaction between the gut microbiota and the mammalian host mediated by metabolism, recent papers have continued to show that immunologic interactions between the host and microbiota are intricate and important. An example of this type of relationship was highlighted in a recent study that examined how innate immune responses could shape the microbial ecology of the gut and how this in turn influenced the development of disease susceptibility and host. Investigators showed that mice that were deficient in the function of the NLRP 6 inflammasome (a sensor of damage-associated molecular patterns, including those produced by enteropathogenic microbes) possessed a gut microbiota that was distinct from wild-type mice $\left(14^{* *}\right)$. Furthermore, these mice were more susceptible to severe colitis when exposed to dextran sodium sulfate (DSS). Interestingly, this susceptibility was shown to be due to the altered microbial community as transfer of this community to wildtype mice (via cohousing) increased the susceptibility of wild-type mice to DSS.

While multiple previous studies have shown that the host immune system has a role in shaping the structure of the human microbiota, recent studies have also looked at the reverse. A number of studies have examined the ability of the indigenous microbiota to direct host immune responses, presumably to the benefit of both parties. Regulatory T cells are critical in maintaining gastrointestinal homeostasis by regulating inflammatory responses. Defects in regulatory $\mathrm{T}$ cells are thought to contribute to the pathogenesis of a variety of diseases where an inappropriate inflammatory response is encountered such as in inflammatory bowel disease. Two recent papers examined the role members of the gastrointestinal microbiota play in the induction of colonic regulatory $\mathrm{T}$ cells. It was shown that colonization of germ-free mice with a complex microbial community derived from SPF mice increased the numbers of regulatory $\mathrm{T}$ cells in the colonic lamina propria. One group demonstrated that a cocktail of 46 strains of the genus Clostridium (primarily from clusters IV and XIVa) would also result in the induction of colonic regulatory $\mathrm{T}$ cells in previously germ-free mice $\left(15^{*}\right)$. Interestingly, colonization with a mixture of 3 Lactobacillus strains or with segmented-filamentous bacteria (SFB, which have been shown to induce TH 17 responses) did not affect the numbers of colonic regulatory T cells. Another group used a cocktail of 8 organisms known as the altered Schaedler flora to colonize germ-free mice. Mice colonized with the altered Schaedler Flora also developed colonic regulatory T cell responses $\left(16^{*}\right)$. Both groups showed that this induction of regulatory $\mathrm{T}$ cells was protective to the host as colonized animals developed less severe colonic inflammation when treated with the DSS.

A 3rd group examined the interplay between the adaptive immune system and the microbiota by examining colonization of mice with Bacteroides fragilis. This group has previously shown that a polysaccharide antigen (PSA) in $B$. fragilis served as a "symbiosis factor" for this organism. Mono colonization of germ-free mice with $B$. fragilis did not induce pro-inflammatory $\mathrm{T}$ helper $17\left(\mathrm{~T}_{\mathrm{H}} 17\right)$ cell development $\left(17^{*}\right)$. However mono colonization with a PSA-deficient $B$. fragilis strain resulted in significant $\mathrm{T}_{\mathrm{H}} 17$ responses in the gut. Purified PSA could suppress these $\mathrm{T}_{\mathrm{H}} 17$ responses to the PSA-deficient $B$. fragilis strain. This group then investigated the mechanisms by which $B$. fragilis suppressed inflammatory $\mathrm{T}_{\mathrm{H}} 17$ responses. They found that regulatory $\mathrm{T}$ cell function was required to 
suppress $\mathrm{T}_{\mathrm{H}} 17$ responses. Furthermore, they found that PSA appeared to signal through tolllike receptor 2 on regulatory $\mathrm{T}$ cells to mediate an IL-10-dependent regulatory response.

The microbiota also has a role in shaping host innate immune responses. A recent study showed that recognition of microbial products produced by the indigenous gut microbiota under basal conditions resulted in priming of the inmate immune system, specifically enhancing neutrophil function $\left(18^{*}\right)$. Bone marrow-derived neutrophils from germ-free animals were less efficient at killing the bacterial pathogens $S$. pneumoniae and $S$. aureus compared to those from previously germ-free animals that were colonized with the complex microbiota. Similar to the findings with $B$. fragilis, this signal from the microbiota was mediated through a pattern-recognition receptor, in this case Nod1. Nod1-deficient bone marrow-derived neutrophils were less efficient at bacterial killing whether or not the animals from which these cells were obtained were germ free or conventionalized.

Taken together these studies indicate that the relationship between the host immune system and the indigenous microbiota is a highly complex and active condition. Adaptation on the part of both the host and the microbe is required in order to maintain a homeostatic relationship. Signals are exchanged in both directions to maintain a mutually beneficial interaction.

\section{Gastrointestinal Disease Associations with The Intestinal Microbiota}

I will now review some key studies that have examined the role of the intestinal microbiota with specific clinical conditions. A number of reports have been published that have looked for associations between the microbiome and various diseases including obesity (19), necrotizing enterocolitis (20), type I and type II diabetes $(21,22)$, irritable bowel syndrome $(23,24)$ and colon cancer $(25)$. However, two major clinical entities will be the focus of this section, inflammatory bowel disease (IBD) and infectious colitis. Both of these conditions have been the focus of a great deal of research that has focused on microbial factors, long before the current interest in microbiome research developed. Additionally, these areas probably have the most attention in terms of the number of investigators and studies that have tried to link functional and mechanistic aspects of the gut microbiome in disease pathogenesis. The increasing rate at which studies investigating associations between disease and the microbiota has necessitated a selection of studies noteworthy in either their scope, or attempt to go beyond statistical associations and include mechanistic insight into the relationship.

\section{Inflammatory Bowel Disease}

As reviewed above, there have been interesting studies using murine systems to examine the relationship between the gut microbiota and the immune system. These studies are often meant to provide insight into IBD. For example, several of the studies reviewed above looked at the response of mice to treatment with DSS as a model of human IBD. I will now review some of the recent studies where the microbiota was studied in the setting of human IBD. A number of studies continue to be published where investigators obtain specimens (often fecal specimens) from patients with IBD and compare the structure of the microbiota to those found in matched controls. Often, dramatic differences are found, but is difficult to understand what the significance of these associations are. The majority of these studies are confounded by the treatments that IBD patients receive, differences in host factors including genotype and many involve small numbers of subjects. In an attempt to try and limit the effects of host genotype on the microbiota, a number of twin studies have been conducted where monozygotic and dizygotic twins that are either concordant or discordant for IBD and their gut microbiota profiled. One study used pyrotag sequencing to examine the fecal samples from a cohort of 40 twin pairs concordant or discordant for both Crohn's disease 
(CD) or ulcerative colitis (UC) (26*). Patients with CD (either colonic or ileal) had distinct microbiota profiles compared to healthy controls or patients with ulcerative colitis. Furthermore, the fecal microbiota in patients with ileal CD differed from that in patients with predominantly colonic disease. Interestingly, the patients with UC had a gut microbiome that was indistinguishable from that in controls. Specific bacteria appeared to be either increased or decreased in relative abundance with each of the IBD disease phenotypes, although a specific role in the pathogenesis of IBD could not be assigned in such a study. The finding that patients with UC did not appear to have a distinct microbiome signature compared to controls contrasts with the results of another twin study $\left(27^{*}\right)$. These investigators examined sigmoid biopsies from patients with UC (in contrast to the fecal samples employed by the previous study) and found a unique microbiota profile in UC patients compared to their unaffected twin controls. This group also examined the host response to this altered microbiota by determining mRNA profiles by microarray analysis. In patients with $\mathrm{UC}$, transcripts related to oxidative and immune responses were differentially expressed compared to healthy twins. Although the results from these two twin studies are intriguing it remains to be determined what the actual linkage is between the altered microbiota profiles encountered and the disease phenotype. Such studies, due to fact that the patients all have disease at the time of microbiota analysis make it difficult to distinguish between association and causality. It is likely that followup studies will require both additional human clinical surveys, but to approach mechanisms, complementary animal studies will allow direct tests of causality.

Besides using twins to overcome the problem of genetic heterogeneity, another approach is to have each patient act as their own control. Another study of the role of the microbiota in IBD compared the mucosa-associated microbiota in inflamed regions of the gut of patients with $\mathrm{CD}$ and UC to non-inflamed regions from these same patients and in healthy controls (28). Compared to controls, the mucosal microbiota from patients with IBD was distinct in community structure. As in the first twin study above, this was more apparent in the patients with CD compared to controls as opposed to the UC patients. Additionally, these investigators report that there were distinct differences between the inflamed and noninflamed portions of the intestine in patients with IBD. It should be noted however, that these intra-individual differences were generally smaller than the inter-individual differences in the gut microbiota.

\section{Infectious Colitis}

For over a century the view as to how microbes played a causative role in disease has been influenced by the concepts proposed by Robert Koch. Koch's postulates gives rise to the impression that infection with a single pathogenic microbe is sufficient to cause disease. Recently, many investigators are beginning to re-examine this dogma and are investigating how the tripartite interaction between pathogen, host and the indigenous microbiota influences the development of disease.

Gastroenteritis due to Salmonella has been well studied in terms of the genetic adaptations the pathogen has evolved and the role of the host immune system in determining disease outcome. Two recent studies have examined how interactions with the intestinal microbiota influences resultant Salmonella infection. One group employed antibiotic treatment to alter the indigenous microbiota and then experimentally infected these antibiotic-treated mice with $S$. Typhimurium $(29 *)$. All 3 antibiotics employed (streptomycin, vancomycin and metronidazole) altered the indigenous microbiota and increased the ability of $S$. Typhimurium to colonize the intestine. However while greater inflammation was seen in the intestinal tract of streptomycin or vancomycin-treated animals infected with $S$. Typhimurium compared to infected animals that did not receive any antibiotics, no 
significant pathology was seen in metronidazole-treated animals that were similarly challenged with $S$. Typhimurium. This indicates that specific subsets of the intestinal microbiota may mediate protection against the development of colitis. Culture-independent comparison of the microbiota remaining in animals treated with the various antibiotics was performed but the significance of these differences needs to be directly tested in follow-up experiments. Another elegant study examined how salmonella is able to take advantage of the inflammatory response generated by the host to promote its own survival within the gut microbial community (30**). It had been known for many decades that salmonella is able to use the compound tetrathionate as a terminal electron acceptor. This fact is used in the clinical microbiology lab to perform culture enrichment of salmonella from fecal samples. These investigators determined that hydrogen sulfide, which is produced in relatively large quantities by certain members of the indigenous gut microbiota is detoxified by the intestinal mucosa by conversion to thiosulfate. Thiosulfate is in turn oxidized by products produced by infiltrating neutrophils, which characterize the acute inflammatory response to salmonella infection. These findings highlight another example of the intricate interactions that take place in the gut. In this case, the pathogen takes advantage of the hosts inflammatory response to its presence to create an environment that allows it to outcompete per resident microbes.

Competition between potential pathogens and the indigenous microbiota is a common theme that is emerging in the study of infectious colitis. Similar to the situation described above for salmonella, vancomycin-resistant enterococcus has been shown to trigger post production of antimicrobial peptides that decreases the resistance of the mouse intestine to VRE colonization presumably by altering the community structure of the resident microbiota (31). In a follow-up study, these investigators used culture independent profiling of the intestinal microbiota to determine the specific changes that antibiotic treatment triggered $(32 *)$. They noted that changes triggered by specific antibiotics could persist following cessation of the drug and these altered communities remained susceptible to VRE colonization. In susceptible communities VRE would become the dominant organism in the gut. Most interestingly, they extended these animal studies by examining patients who developed VRE bacteremia following bone marrow transplant. Patients who developed VRE bacteremia were found to have domination of their gut microbiota by the organism prior to the development of bloodstream infection. This result suggests that profiling the intestinal microbiota of patients may have a future role in guiding subsequent therapy.

Our group as investigated the role of the indigenous microbiota in protecting the host against infection with the toxin-producing bacterium Clostridium difficile. We recently published a study where we related specific changes in the indigenous microbiota, brought on by antibiotic administration, with susceptibility to colonization with $C$. difficile and the severity of subsequent disease (33). Again, are findings support the idea that there is a dynamic interplay between potential pathogens and the indigenous microbiota. Patients with $C$. difficile infection are generally treated with in antibiotic regimen targeting the pathogen. Recently however, is been noted that a greater proportion of patients with $C$. difficile infection will develop recurrent disease with symptoms returning after specific $C$. difficile antibiotic therapy is stopped. Several groups including our own have reported that recurrent C. difficile is in at least part due to abnormal diversity of the remaining indigenous microbiota. Such results underscore the rationale behind a seemingly unpalatable yet relatively successful treatment for refractory $C$. difficile infection, the transplantation of fecal material from a healthy individual to the patient with recurrent infection. Two recent reports have shown that treatment with donor feces results in long-term changes in the microbial community present in the intestine of the recipient $\left(34^{*}, 35^{*}\right)$. 


\section{Conclusions}

This is an exciting time for investigators that are interested in understanding the complex interaction between mammalian hosts and their microbial partners. Advances in technology have made this previously daunting task more approachable. This has led to an explosion of studies that have investigated associations between the gut microbiota and various diseases states. In spite of the significant effort that has been expended in discovering these associations we are just at the early stages of revealing the mechanisms by which changes in microbial communities can disrupt gastrointestinal homeostasis. Future work that uncovers the principles by which gut microbial communities contribute to intestinal health could lead to novel preventative strategies and therapies for a variety of gastrointestinal conditions.

\section{Acknowledgments}

This work was supported in part by NIH grants DK070875 and AI090871

\section{References cited}

1. Cardenas E, Tiedje JM. New tools for discovering and characterizing microbial diversity. Curr Opin Biotechnol. 2008 Dec; 19(6):544-9. [PubMed: 18984052]

2. Roh SW, Abell GC, Kim KH, Nam YD, Bae JW. Comparing microarrays and next-generation sequencing technologies for microbial ecology research. Trends Biotechnol. 2010 Jun; 28(6):291-9. [PubMed: 20381183]

3. Robinson CJ, Bohannan BJ, Young VB. From structure to function: the ecology of host-associated microbial communities. Microbiol Mol Biol Rev. 2010 Sep; 74(3):453-76. [PubMed: 20805407]

4. Peterson J, Garges S, Giovanni M, McInnes P, Wang L, Schloss JA, et al. The NIH Human Microbiome Project. Genome Res. 2009 Dec; 19(12):2317-23. [PubMed: 19819907]

**5. Arumugam M, Raes J, Pelletier E, Le Paslier D, Yamada T, Mende DR, et al. Enterotypes of the human gut microbiome. Nature. 2011 May 12; 473(7346):174-80. This study used a large, internation dataset to determine that the gut microbial communities from individuals segregate into 3 distinct clusters, termed "enterotypes." The functional and clinical relevence of these enterotypes to their hosts is likely to be an area of intense interest in the coming years. [PubMed: 21508958]

*6. Benson AK, Kelly SA, Legge R, Ma F, Low SJ, Kim J, et al. Individuality in gut microbiota composition is a complex polygenic trait shaped by multiple environmental and host genetic factors. Proc Natl Acad Sci U S A. 2010 Nov 2; 107(44):18933-8. This study identified specific genetic loci that were linked to the presence of specific members of the gut microbial community. [PubMed: 20937875]

7. Antonopoulos DA, Huse SM, Morrison HG, Schmidt TM, Sogin ML, Young VB. Reproducible community dynamics of the gastrointestinal microbiota following antibiotic perturbation. Infect Immun. 2009 Jun; 77(6):2367-75. [PubMed: 19307217]

*8. Dethlefsen L, Relman DA. Incomplete recovery and individualized responses of the human distal gut microbiota to repeated antibiotic perturbation. Proc Natl Acad Sci U S A. 2011 Mar 15; 108(Suppl 1):4554-61. This study demonstrated that short term antibiotic administration could have extensive and long-term effects on the structure of the gut microbiome. [PubMed: 20847294]

*9. Antunes LC, Han J, Ferreira RB, Lolic P, Borchers CH, Finlay BB. Effect of antibiotic treatment on the intestinal metabolome. Antimicrob Agents Chemother. 2011 Apr; 55(4):1494-503. A demonstration of the wide ranging effects that antibiotics can have on the metabolic function of the microbiome. [PubMed: 21282433]

*10. Swann JR, Tuohy KM, Lindfors P, Brown DT, Gibson GR, Wilson ID, et al. Variation in antibiotic-induced microbial recolonization impacts on the host metabolic phenotypes of rats. $\mathrm{J}$ Proteome Res. 2011 Aug 5; 10(8):3590-603. This study showed that changes in the gut microbiome could affect systemic metabolic activity. [PubMed: 21591676] 
**11. Donohoe DR, Garge N, Zhang X, Sun W, O’Connell TM, Bunger MK, et al. The microbiome and butyrate regulate energy metabolism and autophagy in the mammalian colon. Cell Metab. 2011 May 4; 13(5):517-26. The authors demonstrated that the energy status of colonocytes was strongly influenced by the microbiota, with colonocytes from germ-free mice existing in an energy -deprived state. This study provides direct evidence that the microbial community, as a whole, can provide great benefit to the host. [PubMed: 21531334]

12. Turnbaugh PJ, Ridaura VK, Faith JJ, Rey FE, Knight R, Gordon JI. The effect of diet on the human gut microbiome: a metagenomic analysis in humanized gnotobiotic mice. Sci Transl Med. 2009 Nov 11.1(6):6ra14.

13. Zivkovic AM, German JB, Lebrilla CB, Mills DA. Human milk glycobiome and its impact on the infant gastrointestinal microbiota. Proc Natl Acad Sci U S A. 2011 Mar 15; 108( Suppl 1):4653-8. [PubMed: 20679197]

**14. Elinav E, Strowig T, Kau AL, Henao-Mejia J, Thaiss CA, Booth CJ, et al. NLRP6 inflammasome regulates colonic microbial ecology and risk for colitis. Cell. 2011 May 27; 145(5):745-57. Evidence for the two-way communication between host and microbiota that establishes gut homeostasis. The gut microbiome is shaped by the host, and in turn provides signals that diminish pathogenic inflammatory responses in the setting of epithelial damage. [PubMed: 21565393]

*15. Atarashi K, Tanoue T, Shima T, Imaoka A, Kuwahara T, Momose Y, et al. Induction of colonic regulatory T cells by indigenous Clostridium species. Science. 2011 Jan 21; 331(6015):337-41. This study and the next demonstrate the regulatory adaptive immune responses are generated in response to non-pathogenic gut microbes. [PubMed: 21205640]

*16. Geuking MB, Cahenzli J, Lawson MA, Ng DC, Slack E, Hapfelmeier S, et al. Intestinal bacterial colonization induces mutualistic regulatory T cell responses. Immunity. 2011 May 27; 34(5): 794-806. [PubMed: 21596591]

*17. Round JL, Lee SM, Li J, Tran G, Jabri B, Chatila TA, et al. The Toll-like receptor 2 pathway establishes colonization by a commensal of the human microbiota. Science. 2011 May 20; 332(6032):974-7. This study examines mechanisms by which members of the indigenous microbiota can signal to the host in order to maintain colonization of the gut. [PubMed: 21512004]

*18. Clarke TB, Davis KM, Lysenko ES, Zhou AY, Yu Y, Weiser JN. Recognition of peptidoglycan from the microbiota by Nod1 enhances systemic innate immunity. Nat Med. 2010 Feb; 16(2): 228-31. This study demonstrates role that gut microbes can have on priming systemic innate responses to bacterial pathogens. [PubMed: 20081863]

19. Greiner T, Backhed F. Effects of the gut microbiota on obesity and glucose homeostasis. Trends Endocrinol Metab. 2011 Apr; 22(4):117-23. [PubMed: 21353592]

20. Mai V, Young CM, Ukhanova M, Wang X, Sun Y, Casella G, et al. Fecal microbiota in premature infants prior to necrotizing enterocolitis. PLoS One. 2011; 6(6):e20647. [PubMed: 21674011]

21. Giongo A, Gano KA, Crabb DB, Mukherjee N, Novelo LL, Casella G, et al. Toward defining the autoimmune microbiome for type 1 diabetes. ISME J. 2011 Jan; 5(1):82-91. [PubMed: 20613793]

22. Larsen N, Vogensen FK, van den Berg FW, Nielsen DS, Andreasen AS, Pedersen BK, et al. Gut microbiota in human adults with type 2 diabetes differs from non-diabetic adults. PLoS One. 2010; 5(2):e9085. [PubMed: 20140211]

23. Carroll IM, Ringel-Kulka T, Keku TO, Chang YH, Packey CD, Sartor RB, et al. Molecular Analysis of the Luminal and Mucosal-Associated Intestinal Microbiota in Diarrhea-Predominant Irritable Bowel Syndrome. Am J Physiol Gastrointest Liver Physiol. 2011 Jul 7.

24. Saulnier DM, Riehle K, Mistretta TA, Diaz MA, Mandal D, Raza S, et al. Gastrointestinal Microbiome Signatures Of Pediatric Patients With Irritable Bowel Syndrome. Gastroenterology. $2011 \mathrm{Jul} 7$.

25. Sobhani I, Tap J, Roudot-Thoraval F, Roperch JP, Letulle S, Langella P, et al. Microbial dysbiosis in colorectal cancer (CRC) patients. PLoS One. 2011; 6(1):e16393. [PubMed: 21297998]

*26. Willing BP, Dicksved J, Halfvarson J, Andersson AF, Lucio M, Zheng Z, et al. A pyrosequencing study in twins shows that gastrointestinal microbial profiles vary with inflammatory bowel disease phenotypes. Gastroenterology. 2010 Dec; 139(6):1844-54. e1. A large twin study that 
demonstrated distinct microbial community structures in patients with Crohn's disease. [PubMed: 20816835]

*27. Lepage P, Hasler R, Spehlmann ME, Rehman A, Zvirbliene A, Begun A, et al. Twin study indicates loss of interaction between microbiota and mucosa of patients with ulcerative colitis. Gastroenterology. $2011 \mathrm{Jul}$; 141(1):227-36. A twin study that showed differential host responses to altered gut communities in UC patients. [PubMed: 21621540]

28. Walker AW, Sanderson JD, Churcher C, Parkes GC, Hudspith BN, Rayment N, et al. Highthroughput clone library analysis of the mucosa-associated microbiota reveals dysbiosis and differences between inflamed and non-inflamed regions of the intestine in inflammatory bowel disease. BMC Microbiol. 2011; 11:7. [PubMed: 21219646]

*29. Ferreira RB, Gill N, Willing BP, Antunes LC, Russell SL, Croxen MA, et al. The intestinal microbiota plays a role in Salmonella-induced colitis independent of pathogen colonization. PLoS One. 2011; 6(5):e20338. A study that demonstrated that specific changes to the microbiota could have differential effects on the priming of host responses to enteric pathogens. [PubMed: 21633507]

**30. Winter SE, Thiennimitr P, Winter MG, Butler BP, Huseby DL, Crawford RW, et al. Gut inflammation provides a respiratory electron acceptor for Salmonella. Nature. 2010 Sep 23; 467(7314):426-9. A study that revealed a complex interaction in the gut whereby the products of anaerobic bacteria are detoxified by the gut epitheliuim and then converted by the metabolic activity associated with inflammation to provide a beneficial environment for a gut pathogen. This study underscores the multiscale linkages between the members that constitute the gut ecosystem, both host and microbial. [PubMed: 20864996]

31. Brandl K, Plitas G, Mihu CN, Ubeda C, Jia T, Fleisher M, et al. Vancomycin-resistant enterococci exploit antibiotic-induced innate immune deficits. Nature. 2008 Oct 9; 455(7214):804-7. [PubMed: 18724361]

*32. Ubeda C, Taur Y, Jenq RR, Equinda MJ, Son T, Samstein M, et al. Vancomycin-resistant Enterococcus domination of intestinal microbiota is enabled by antibiotic treatment in mice and precedes bloodstream invasion in humans. J Clin Invest. 2010 Dec 1; 120(12):4332-41. Demonstration that the status of the gut microbiome may have utility in predicting the risk of bacteremia with enteric pathogens. [PubMed: 21099116]

33. Reeves AE, Theriot CM, Bergin IL, Huffnagle GB, Schloss PD, Young VB. The interplay between microbiome dynamics and pathogen dynamics in a murine model of Clostridium difficile Infection. Gut Microbes. 2011 May 1.2(3)

*34. Khoruts A, Dicksved J, Jansson JK, Sadowsky MJ. Changes in the composition of the human fecal microbiome after bacteriotherapy for recurrent Clostridium difficile-associated diarrhea. J Clin Gastroenterol. 2010 May-Jun;44(5):354-60. This study and the following profile the changes in the gut microbiome brought on by therapeutic administration of feces in order to restore a more normal microbial community. [PubMed: 20048681]

*35. Grehan MJ, Borody TJ, Leis SM, Campbell J, Mitchell H, Wettstein A. Durable alteration of the colonic microbiota by the administration of donor fecal flora. J Clin Gastroenterol. 2010 Sep; 44(8):551-61. [PubMed: 20716985] 


\section{Key Points}

- The gut microbiome plays a key role in maintaining gut homeostasis through interactions with the host immune system and metabolism.

- Alterations in the structure of the gut microbial community have been associated with a large number of disease states, but our understanding of the mechanisms by which these changes results in disease is far from complete.

- Direct manipulation of the gut microbiota may prove to be a novel method for the prevention and treatment of a variety of gastrointestinal disorders. 